\title{
Fully-Automated Chip-Based Nanoelectrospray Tandem Mass Spectrometry of Gangliosides from Human Cerebellum
}

\author{
Alina Zamfir, Željka Vukelić, ${ }^{*}$ Laura Bindila, and Jasna Peter-Katalinić \\ Institute for Medical Physics and Biophysics, Biomedical Analysis, University of Münster, Münster, Germany
}

Reinaldo Almeida, Alistair Sterling, and Mark Allen

Advion BioSciences, Ltd., Norwich, United Kingdom

\begin{abstract}
The introduction of chip-based electrospray (ESI) ion sources into biological mass spectrometry (MS) addressed the fundamental issue of how to analyze minute amounts of complex biological systems. The automation of sample delivery into the MS combined with the chip-based ESI allows for high quality bioanalysis in a high-throughput fashion. These advantages have already been demonstrated in proteomics, direct screening of drugs and drug discovery. As part of our continuing effort to implement automated chip-based mass spectrometry into the field of complex carbohydrate analysis, we hereby report the development of a chipESI MS and MS/MS methodology for the screening of gangliosides. A strategy to characterize a complex ganglioside mixture from human cerebellar tissue, by automated ESIchip-quadrupole time-of-flight (QTOF) MS and MS/MS is presented here. The feasibility of this method, and the general experimental requirements for automated chipESI MS analysis of these carbohydrate species is described. (J Am Soc Mass Spectrom 2004, 15, 1649-1657) (C) 2004 American Society for Mass Spectrometry
\end{abstract}

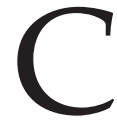
onsiderable efforts are being invested into both the development of automated and miniaturized analytical tools, and their introduction into routine bioscience [1, 2]. Robotic micro- and nano-systems, developed for high-throughput accurate analyses, enable low sample consumption, by limiting losses through handling. This can aid considerable in reducing sample costs and experimental time.

In biological mass spectrometry (MS), the recent introduction of chip-based electrospray (ESI) ion sources has enabled the issues of sensitivity in the analysis of minute amounts of biological materials to be addressed. These ion sources have also enabled automated sample delivery into MS instruments, without risk of cross-contamination, in a high throughput fashion. The advantages of an automated chip-based ESI infusion have already been widely proven in proteomics, direct screening of drugs and drug discovery [1-7].

Carbohydrates, which are an important and ubiquitous class of biopolymers, have been shown to require their own particular analytical conditions, for ESI MS and other related hyphenated techniques. These are

Published online September 25, 2004

Address reprint requests to Dr. A. D. Zamfir, Institute for Medical Physics and Biophysics, Biomedical Analysis, University of Münster, Robert Koch Strasse 31, D-48149 Münster, Germany. E-mail: zamfir@uni-muenster.de

* Also at the Department of Chemistry and Biochemistry, Faculty of Medicine, University of Zagreb, Zagreb, Croatia. quite distinct to those required by proteins and nucleic acids [8-16]. Carbohydrates, apart from having a polyfunctional molecular structure consisting of monosaccharide building blocks, a high diversity and microheterogeneity, also exhibit rather restrictive properties for ion formation and detection, which are variable between the different carbohydrate categories. Our studies are focused on developing new MS strategies to provide a high throughput and sensitive structural analysis of complex carbohydrate mixtures, from biological sources. We have recently described, for the first time to our knowledge, an automated ESIchip-quadrupole time-of-flight (QTOF) MS and tandem MS system for the screening of complex mixtures of oligosaccharides and glycopeptides. These samples originated from the urine of patients suffering from Schindler's disease or from congenital disorder of glycosylation (CDG) [17]. The chip-based ESI MS methodology has demonstrated the potential to detect low abundance, known carbohydrate species, and novel carbohydrate variants from heterogeneous glycopeptide mixtures previously only accessible to structural investigation by combined capillary electrophoresis/mass spectrometry (CE/MS) [18].

Gangliosides, sialylated glycosphingolipids (GSLs), are composed of mono- to poly-sialylated oligosaccharide chains of variable length, attached to the ceramide portion, with variable composition with respect to the types of sphingoid base and fatty acid residues. Gan- 
gliosides are plasma-membrane components, enriched in the microdomains, particular abundant in the central nervous system. Here they participate in cell-to-cell recognition, communication and cell signaling, and are consequently either modulating or triggering various biological events $[19,20]$. Ganglioside composition is dependant on both species and cell type. Specific changes occur during brain development, maturation, and aging, and due to disease or neurodegeneration processes [21-23]. They are therefore regarded as tissue stage- and/or diagnostic markers, and therefore potentially as therapeutic agents. In the human brain, differences in ganglioside composition, quantity, distribution and cell surface expression have been shown to have regional specificity. These differences have been demonstrated by primarily thin-layer chromatography, but also by immunochemical and immunohistochemical methods [24, 25].

The cerebellum, a morpho-anatomically and functionally highly specialized region of the brain, has some characteristic differences in the composition of its major ganglioside species, when compared to those regions of the cerebrum that have been studied [22]. The function, behavior and even survival of cerebellar neurons are highly dependant on the cellular expression of the ganglioside species [26].

To analyze complex ganglioside mixtures from biological sources by MS methods, by either ESI or matrix assisted laser desorption/ionization (MALDI), specific ionization conditions are required. These are necessary to provide a reliable assessment of the sample heterogeneity, and to enable identification of the molecular architecture.

We recently reported an optimized procedure for (-)nanoESI-QTOF MS and tandem MS for the characterization of a native ganglioside mixture from brain tissue [27-30]. This procedure requires elevated parameter values for ionization, and an extended time for MS/MS signal acquisition, which suggests that relatively high sample consumption is needed for identification and structural elucidation.

We have also developed an approach that combines $\mathrm{CE}$ for the separation, with a nano ESI-QTOF MS for detection of single molecular species, and MS/MS for their identification by sequencing [31]. This approach allows for a rapid separation procedure followed by sensitive detection and structural analysis of ganglioside/glycosphingolipid species present in complex mixtures isolated from biological tissues. All MS parameters were found to require optimization, as they all had a significant effect on both MS detection and product ion abundance in fragmentation experiments, when analyzing single ganglioside molecular species from CE eluted fractions. Analyte/CE buffer clusters are optimally decomposed with high capillary and cone voltages in the ESI ion source. These parameters, at high values, however can induce in-source fragmentation jeopardizing the detection of intact molecular ions in MS1, the selection of precursor ions for fragmentation, and result in a long acquisition time to counterbalance the in-source decrease in molecular ion abundance. As part of our effort to implement fully automated chipbased mass spectrometry in the field of complex carbohydrate analysis, a general methodology for the screening of glycosphingolipids was initiated. The methods were optimized with respect to ionization, sensitivity, automated sequencing, speed of analysis, and limited sample consumption. The automated ESIchip-QTOF MS and MS/MS presented here is optimized in negative ion mode, for the characterization of a complex ganglioside mixture from human cerebellar tissue. The data will demonstrate its general utility in ganglioside analysis, and its advantages when compared to capillary-based ESI MS and MS/MS.

\section{Experimental}

\section{Biological Material Sampling and Isolation of Ganglioside Mixture from Human Cerebellar Gray Matter}

A normal adult human (20 years old) cerebellum, without any pathological signs according to morphoanatomical and histopathological examination, originated from a healthy subject who died in a traffic accident. The cerebellum was obtained from the Department of Forensic Medicine, Faculty of Medicine, University of Zagreb, Croatia. Permission for experiments with human tissue for scientific purposes was obtained from The Ethical Commission of the University of Zagreb, Croatia, under project no. 108120 by the Ministry of Science and Technology of the Republic of Croatia.

The cerebellar gray matter was carefully separated from the white matter by dissection. Samples were weighed and stored at $-20{ }^{\circ} \mathrm{C}$ until the extraction procedure. The native mixture of gangliosides was extracted and purified in our laboratories as described in detail elsewhere [27]. The sample was dried in a Speed Vac SPD 111V system (Savant, Düsseldorf, Germany). For ESI MS analysis, a stock solution of the sample at approximately $1 \mathrm{mg} / \mathrm{ml}$ was prepared by dissolving the dried material in methanol. This stock solution was stored at $-20^{\circ} \mathrm{C}$. Dilution of the stock solution in pure methanol yielded the working aliquot concentrations of approximately $15 \mathrm{pmol} / \mu \mathrm{l}$ and 2-3 $\mathrm{pmol} / \mu \mathrm{l}$. Methanol was obtained from Merck (Darmstadt, Germany) and used without further purification. Distilled and deionized water (Mili-Q Water Systems Millipore, Bedford, MA) was used for the preparation of the sample solutions.

\section{Applied Ganglioside Nomenclature}

Gangliosides and their precursor glycosphingolipids are abbreviated according to the system of L. Svennerholm, (J. Neurochem. 1963, 10, 613 and Adv. Exp. Med. Biol. 1980, 125, 11), and the recommendations of the 
IUPAC-IUB Commission on Biochemical Nomenclature (Eur. J. Biochem. 1977, 79, 11 and Eur. J. Biochem. 1998, 257, 293).

\section{Mass Spectrometry}

Mass spectrometry was performed on an orthogonal hybrid quadrupole time-of-flight mass spectrometer (QTOF Micromass, Manchester, U.K.) in the Micromass Z-spray geometry. The QTOF mass spectrometer was interfaced to a PC computer running MassLynx software to control the instrument, acquire and process MS data. The source block temperature was kept at $80^{\circ} \mathrm{C}$. All mass spectra were acquired in the negative ion mode, which is known to be advantageous for ganglioside MS analysis [27, 30, 32]. Tandem mass spectrometry was carried out by automatic selection and fragmentation of the precursor ion with switching from MS to MS/MS (auto MS/MS) in data-dependant analysis mode as described by us elsewhere [18]. CID at low energies was performed using Ar as a collision gas. All mass spectra were calibrated using sodium iodide as a calibrant.

\section{Automated Chip-Based Nanoelectrospray}

Fully automated chip-based nanoelectrospray was performed on a NanoMate 100 incorporating ESI Chip technology (Advion BioSciences, Ithaca, NY) mounted to the QTOF mass spectrometer. The robot was controlled and manipulated by ChipSoft software operating under Windows. The position of the electrospray chip was adjusted with respect to the sampling cone to ensure optimal transfer of the ionic species into the mass spectrometer. In order to prevent any contamination, a glass coated microtiter plate was used for all experiments. $5 \mu \mathrm{l}$ aliquots of the working sample solutions were loaded into a 96-well plate. The robot was programmed to aspirate the sample, followed by $2 \mu \mathrm{l}$ of air into the pipette tip, and to deliver the sample to the inlet side of the microchip. The microchip consists of a $10 \times 10$ array of nozzles etched into the planar surface of a silicon wafer and is fabricated using a variety of techniques, including deep reactive ion etching. The preparation of the microchip is described in detail elsewhere [33]. A channel extends from the nozzle through the microchip to an inlet on the opposite surface and during analysis, the conductive pipette tip containing the sample is engaged against this inlet forming a pressure seal. Each nozzle has an internal diameter of $9 \mu \mathrm{m}$. Electrospray was initiated by applying voltages between 1.45 to $1.67 \mathrm{kV}$ and a head pressure of 0.3 to 0.45 p.s.i. Following sample infusion and MS analysis, the pipette tip was ejected and a fresh tip and nozzle were used for each sample, thus preventing any cross-contamination or carry-over.

\section{Results and Discussion}

Optimization of the Automated Chip-Based
NanoESI-QTOF MS for Ganglioside
Ionization/Detection

The ganglioside sample, G20y, was dissolved in pure methanol to a final concentration of approximately 15 $\mathrm{pmol} / \mu \mathrm{l}$, and transferred into a glass-coated well in the 96-well plate. Chip-based electrospray ionization is initiated with the application of $1.55 \mathrm{kV}$ pipette tip voltage and 0.3 p.s.i. back pressure to the ESI Chip, and $45 \mathrm{~V}$ sampling cone potential. These parameters generate a sustained and consistent spray, which shows a high ionization yield. Figure 1a shows the (-)nanoESIchip-QTOF mass spectrum combined over $1 \mathrm{~min}$ of the total ion current (TIC). Three major ganglioside species are detected here: GM1, GD1, GT1 containing one, two, and three sialic acid moieties, respectively, and each exhibiting two different molecular forms attributable to the fatty acid heterogeneity in the ceramide portion. Due to the mild ESI Chip and sampling cone parameters, certain ions are formed preferentially. These are triply charged ions at $m / z 708.68$ and 718.00, which correspond to two triply sialylated GT1 molecular species, (d18:1/18:0) and (d18:1/20:0), and abundant doubly charged ions at $m / z 917.37$ and 931.39 . These correspond to the disialylated GD1 species, (d18:1/ 18:0) and (d18:1/20:0). The GT1 (d18:1/18:0) and (d18:1/ 20:0) molecular ions are also observed as intense doubly charged ions at $\mathrm{m} / \mathrm{z} 1073.84$ and 1088.38. The less abundant singly charged ions which correspond to the monosialylated GM1 molecular species (d18:1/18:0) and (d18: 1/20:0) are detected at $m / z \quad 1544.47$ and 1573.49, respectively. In addition, singly charged GD1 molecular ions, at a reduced intensity, are also present in the spectrum at $m / z 1857.46$ and 886.41. These ions are related to ganglioside species containing the (d18:1/18:0) and (d18: 1/20:0) ceramide residues, respectively. Using the same G20y sample, independent results have also been obtained by thin-layer chromatography and resorcinol staining (data not shown). These results indicate that a higher abundance of GM1 species and a higher degree of heterogeneity than in Figure 1a should be observed. To correlate the expression patterns from the TLC to the ionized species in the mass spectra, the ionization conditions must be systematically explored. The initial step in the optimization of the ESI Chip ionization/detection for all the single ganglioside components in the G20y sample is to acquire MS data at elevated levels of the ESI Chip and sampling cone potentials. This approach has already been demonstrated [30] to enhance the ionization yield of larger ganglioside species from human brain tissues. In Figure $1 b$, the nanoESIchip-QTOF mass spectrum recorded for 1 min at $1.67 \mathrm{kV}$ tip potential and $135 \mathrm{~V}$ sampling cone potential is presented. With these parameters a greater proportion of the GM1 molecules should be ionized and detected. Indeed GM1 (d18:1/18:0) and (d18:1/20:0) are detected as abundant singly charged ions at $\mathrm{m} / \mathrm{z} 1544.47$ and 1572.51 (Figure 1b). According to our experimental 


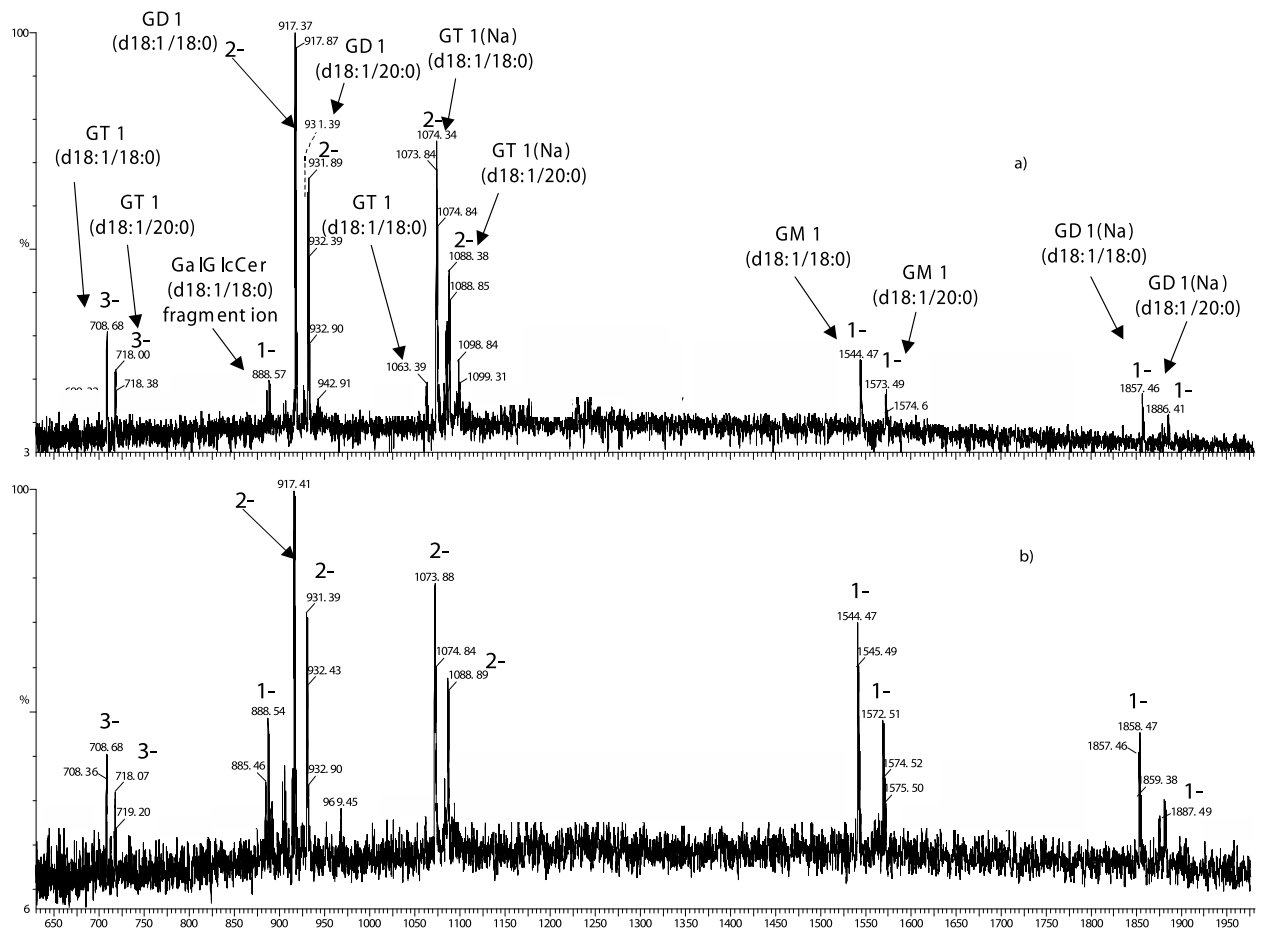

Figure 1. Fully automated (-)nanoESIchip-QTOF MS of the G20y ganglioside mixture from gray matter of normal human cerebellum. Sample concentration $15 \mathrm{pmol} / \mu \mathrm{l}$ in methanol. n.a. = not assigned (a) sampling cone potential $45 \mathrm{~V}$; acquisition time $1 \mathrm{~min}$. (b) sampling cone potential $135 \mathrm{~V}$; acquisition time $1 \mathrm{~min}$.

experience (data not shown) with purified GM1 as well as GD1 fractions, the intensity of the molecular ion increases with rising of cone potential. Nevertheless, in the case of complex mixtures, the ion intensity may be partially related also to increased in-source fragmentation of more complex species.

Up to nine ganglioside variants are detected in this sample under set instrument parameters. Other minor components and/or longer chains, which are assumed to be present in the sample, could not, however, be detected. Of interest is that with the sampling cone potential of $135 \mathrm{~V}$, the charge distribution did not shift significantly towards lower values, as expected. In addition, the signal intensities corresponding to doubly and triply charged ions were not altered. As already mentioned, a potential disadvantage of higher voltages is the in-source fragmentation of ganglioside species. In the case of ESI Chip, the in-source decay was minimal, despite the application of a high cone potential. This is illustrated in Figure 1b, where the signals at $m / z 888.54$ and 906.61, are only slightly increased. These ions correspond to GlcCer ${ }^{-}$(d18:1/18:0) in-source fragments.

Earlier work [7] has demonstrated that the NanoMate ESIchip MS can achieve subpico- and femtomolar levels of sensitivity in peptide analysis. We have reported results [17] with the NanoMate ESIchip-QTOF MS, demonstrating that spectra with a high signal to noise ratio, providing rich structural information, can be obtained from complex glycopeptide mixtures, within a few minutes of signal acquisition. This glycopeptide data was generated from samples with a significantly lower concentration of 3 $\mathrm{pmol} / \mu \mathrm{l}$.

By diluting the G20y sample serially for the nanoESIchip-QTOF MS experiments, the ionization of the ganglioside components was significantly enhanced. Figure $2 \mathrm{a}$ and $\mathrm{b}$ show spectra of the same sample analyzed by ESIchip-QTOF MS. In these experiments, the conditions have been modified as detailed below. The sample has been diluted to $2-3 \mathrm{pmol} / \mu \mathrm{l}$, the ESI tip potential is increased to $1.67 \mathrm{kV}$, and the sample cone potential is varied from 45 to $135 \mathrm{~V}$. A fair signal-to-noise ratio is obtained in less than $1 \mathrm{~min}$, however the mass spectrum is recorded for $3 \mathrm{~min}$ with variable source parameters. This strategy allows for a high ionization yield, and the detection of a larger number of species. Using these conditions (Figure 2), a more realistic representation of the ganglioside heterogeneity, when compared against the TLC pattern, is obtained. It is therefore possible to conclude the following: A well-defined set of experimental conditions allows ganglioside mixture analysis with a high degree of sensitivity, without compromising the ionization of minor biologically important components. Furthermore, by the ESIchip MS approach the in-source decay is avoided, and the analysis time is reduced significantly.

Table 1 lists the assignment of singly, doubly and triply charged ions corresponding to 46 different ganglioside components, which have been identified by automated chip-based nanoESI-QTOF MS. It is clear that the G20y ganglioside mixture is dominated by GD1 glycoforms. Nineteen different doubly charged molecular variants of 


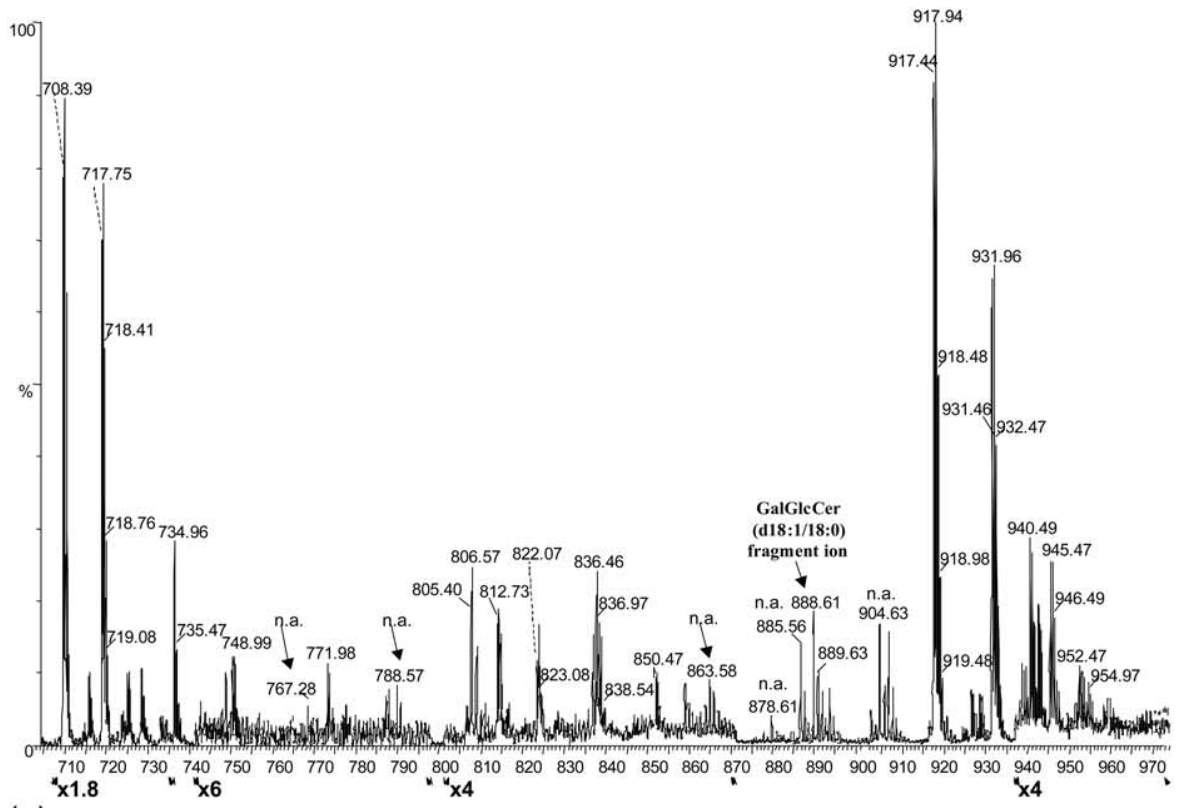

(a)

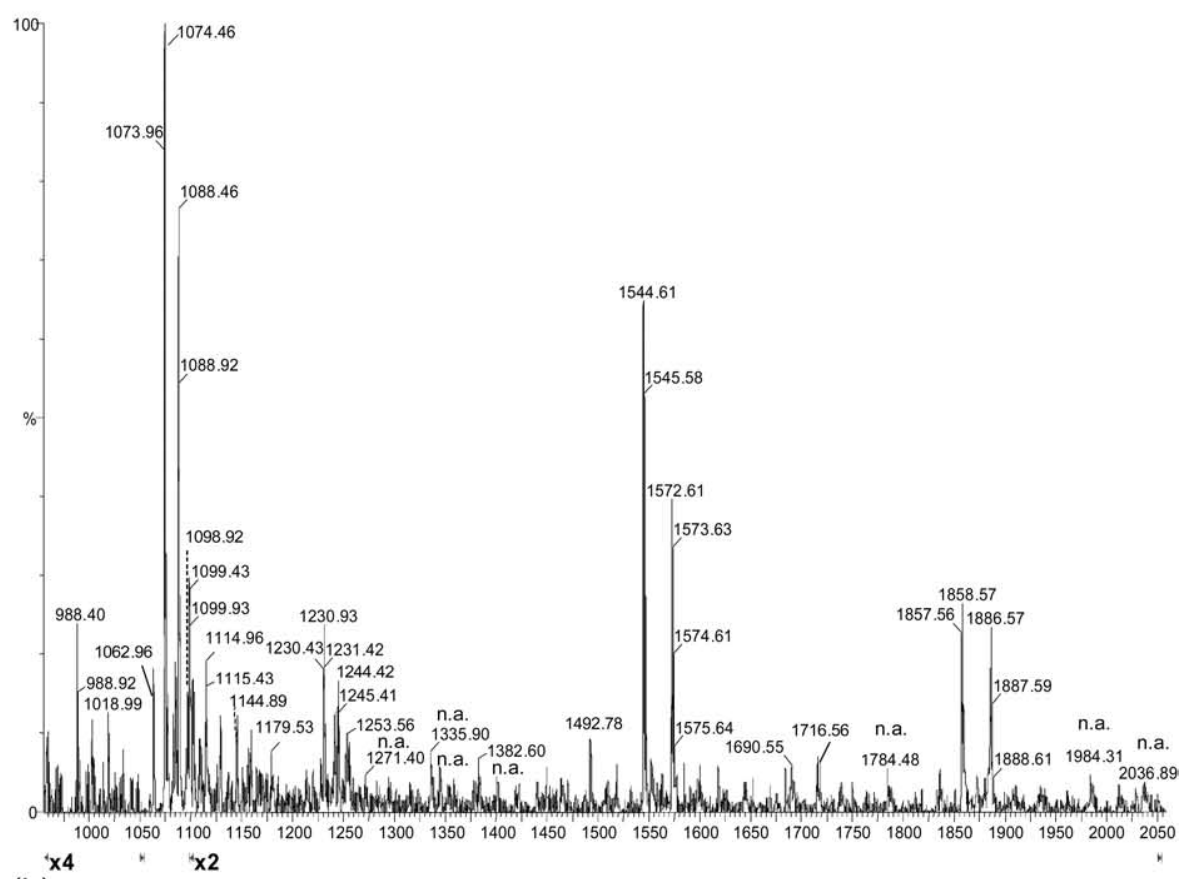

(b)

Figure 2. Fully automated (-)nanoESIchip-QTOF MS of the G20y mixture. Sample concentration 2-3 $\mathrm{pmol} / \mu \mathrm{l}$ in methanol; acquisition time $3 \mathrm{~min}$; sampling cone potential $45-135 \mathrm{~V}$. n.a. $=$ not assigned (a) $m / z(700-980)$. (b) $m / z(980-2050)$.

GD1 have been detected. Also identified in the mixture are GM1, GM2, GM3, GD2, GD3, GT1, and GQ1, expressing different ceramide portions.

The most abundant ions are assigned to doubly charged pseudomolecular species originating from GD1 (d18:1/18:0) and (d18:1/20:0), GT1 ${ }^{-}(\mathrm{d} 18: 1 / 18: 0)$ and (d18:1/20:0) followed by singly charged ions attributed to GM1 (d18:1/18:0) and (d18:1/20:0). GT1 (d18:1/18:0) and (d18:1/20:0) molecular species were detected as intense triply charged ions at $m / z 708.39$ and 717.75 as well.
Biologically important $O$-acetylated and/or fucosylated GM1, GD1, GT1, and GQ1 exhibiting a high degree of heterogeneity in their ceramide motifs are also detected. Fucosyl-GD1 (Table 1) has been shown to have the most glycoforms, and these six fucosylated variants have all been detected as fairly abundant doubly charged ions.

The triply charged ions at $\mathrm{m} / \mathrm{z} 722.39$ and 731.74 are assigned to O-Ac-GT1 (d18:1/18:0) and O-Ac-GT1 (d18:1/ 20:0), respectively, while the doubly charged ions at $\mathrm{m} / \mathrm{z}$ 1128.92, 1144.89, 1159.89 correspond to Fuc-GT1 (d18:1/ 
Table 1. Composition of single components in the G20y ganglioside mixture from gray matter of normal human cerebellum as detected by a fully automated (-)nanoESIchip-QTOF MS

\begin{tabular}{|c|c|c|c|}
\hline \multirow[b]{2}{*}{ Type of molecular ion } & \multicolumn{2}{|c|}{$\mathrm{m} / \mathrm{z}$ (monoisotopic) } & \multirow[b]{2}{*}{ Assigned structure } \\
\hline & Detected & Calculated & \\
\hline$[\mathrm{M}+2 \mathrm{Na}-4 \mathrm{H}]^{2-}$ & 611.40 & 611.35 & GM3 (d18:1/18:0) \\
\hline$[\mathrm{M}-\mathrm{H}]^{-}$ & 1179.57 & 1179.74 & \\
\hline$[\mathrm{M}-\mathrm{H}]^{-}$ & 1382.60 & 1382.82 & GM2 (d18:1/18:0) \\
\hline$[\mathrm{M}-2 \mathrm{H}]^{2-}$ & 734.96 & 734.91 & GD3 (d18:1/18:0) \\
\hline$[\mathrm{M}+\mathrm{Na}-2 \mathrm{H}]^{-}$ & 1492.78 & 1492.81 & \\
\hline$[\mathrm{M}-2 \mathrm{H}]^{2-}$ & 748.99 & 748.93 & GD3 (d18:1/20:0) \\
\hline$[\mathrm{M}-\mathrm{H}]^{-}$ & 1518.51 & 1518.85 & GM1, nLM1 and /or LM1 (d18:0/16:0) \\
\hline$[\mathrm{M}-2 \mathrm{H}]^{2-}$ & 771.98 & 771.93 & GM1, nLM1 and /or LM1 (d18:1/18:0) \\
\hline$[\mathrm{M}-\mathrm{H}]^{-}$ & 1544.61 & 1544.85 & \\
\hline$[\mathrm{M}-2 \mathrm{H}]^{2-}$ & 786.00 & 785.92 & GM1, nLM1 and /or LM1 (d18:1/20:0) \\
\hline$[\mathrm{M}-\mathrm{H}]^{-}$ & 1572.61 & 1572.85 & \\
\hline$[\mathrm{M}-\mathrm{H}]^{-}$ & 1690.55 & 1690.93 & Fuc-GM1(d18:1/18:0) \\
\hline$[\mathrm{M}-\mathrm{H}]^{-}$ & 1716.56 & 1716.94 & Fuc-GM1 (d18:1/20:1) \\
\hline$[\mathrm{M}-2 \mathrm{H}]^{2-}$ & 836.46 & 836.45 & GD2 (d18:1/18:0) \\
\hline$[\mathrm{M}-2 \mathrm{H}]^{2-}$ & 850.47 & 850.47 & GD2 (d18:1/20:0) \\
\hline$[\mathrm{M}-2 \mathrm{H}]^{2-}$ & 917.44 & 917.48 & GD1, nLD1 and /or LD1 (d18:1/18:0) \\
\hline$[\mathrm{M}+\mathrm{Na}-3 \mathrm{H}]^{2-}$ & 928.45 & 928.47 & \\
\hline$[\mathrm{M}-\mathrm{H}]^{-}$ & 1835.62 & 1835.96 & \\
\hline$[\mathrm{M}+\mathrm{Na}-2 \mathrm{H}]^{-}$ & 1857.56 & 1857.95 & \\
\hline$[\mathrm{M}-2 \mathrm{H}]^{2-}$ & 926.44 & 926.48 & GD1, nLD1 and /or LD1 (t18:0/18:0) \\
\hline$[\mathrm{M}-2 \mathrm{H}]^{2-}$ & 924.44 & 924.49 & GD1, nLD1 and /or LD1 (d18:1/19:0) \\
\hline$[\mathrm{M}-2 \mathrm{H}]^{2-}$ & 931.46 & 931.49 & GD1, nLD1 and /or LD1 (d18:1/20:0) \\
\hline$[\mathrm{M}+\mathrm{Na}-3 \mathrm{H}]^{2-}$ & 942.44 & 942.48 & \\
\hline$[\mathrm{M}-\mathrm{H}]^{-}$ & 1885.60 & 1885.98 & \\
\hline$[\mathrm{M}-2 \mathrm{H}]^{2-}$ & 940.49 & 940.50 & GD1, nLD1 and /or LD1 (t18:0/20:0) \\
\hline$[\mathrm{M}-2 \mathrm{H}]^{2-}$ & 938.44 & 938.50 & GD1, nLD1 and /or LD1 (d18:1/21:0) \\
\hline$[\mathrm{M}-2 \mathrm{H}]^{2-}$ & 945.47 & 945.51 & GD1, nLD1 and /or LD1 (d18:1/22:0) \\
\hline$[\mathrm{M}-2 \mathrm{H}]^{2-}$ & 954.46 & 954.51 & GD1, nLD1 and /or LD1 (t18:0/22:0) \\
\hline$[\mathrm{M}-2 \mathrm{H}]^{2-}$ & 952.47 & 952.52 & GD1, nLD1 and /or LD1 (d18:1/23:0) \\
\hline$[\mathrm{M}-2 \mathrm{H}]^{2-}$ & $958.46^{*}$ & 958.52 & GD1, nLD1 and /or LD1 (d18:1/24:1) \\
\hline$[\mathrm{M}-2 \mathrm{H}]^{2-}$ & 966.44 & 966.53 & GD1, nLD1 and /or LD1 (d18:1/25:0) or (d20:1/23:0) \\
\hline$[\mathrm{M}-2 \mathrm{H}]^{2-}$ & 988.40 & 988.49 & Fuc-GD1 (d18:1/18:2) \\
\hline$[\mathrm{M}-2 \mathrm{H}]^{2-}$ & 990.40 & 990.51 & Fuc-GD1 (d18:1/18:0) \\
\hline$[\mathrm{M}-2 \mathrm{H}]^{2-}$ & $999.41 *$ & 999.51 & Fuc-GD1 (t18:0/18:0) \\
\hline$[\mathrm{M}-2 \mathrm{H}]^{2-}$ & 1002.41 & 1002.51 & Fuc-GD1 (d18:1/20:2) \\
\hline$[\mathrm{M}-2 \mathrm{H}]^{2-}$ & 1004.42 & 1004.52 & Fuc-GD1 (d18:1/20:0) \\
\hline$[\mathrm{M}-2 \mathrm{H}]^{2-}$ & $1013.44^{*}$ & 1013.53 & Fuc-GD1 (t18:0/20:0) \\
\hline$[\mathrm{M}-2 \mathrm{H}]^{2-}$ & 1018.99 & 1019.02 & GalNAc-GD1 (d18:1/18:0) \\
\hline$[\mathrm{M}-2 \mathrm{H}]^{2-}$ & $1032.93^{*}$ & 1033.03 & GalNAc-GD1 (d18:1/20:0) \\
\hline$[\mathrm{M}-3 \mathrm{H}]^{3-}$ & 708.39 & 708.35 & GT1 (d18:1/18:0) \\
\hline$[\mathrm{M}-2 \mathrm{H}]^{2-}$ & 1062.96 & 1063.03 & \\
\hline$[\mathrm{M}+\mathrm{Na}-3 \mathrm{H}]^{2-}$ & 1073.96 & 1074.02 & \\
\hline$[\mathrm{M}+2 \mathrm{Na}-4 \mathrm{H}]^{2-}$ & 1084.93 & 1085.01 & \\
\hline$[\mathrm{M}-3 \mathrm{H}]^{3-}$ & 714.41 & 714.35 & GT1 (t18:0/18:0) \\
\hline$[\mathrm{M}+\mathrm{Na}-3 \mathrm{H}]^{2-}$ & 1082.92 & 1083.02 & \\
\hline$[\mathrm{M}-3 \mathrm{H}]^{3-}$ & 717.75 & 717.69 & GT1 (d18:1/20:0) \\
\hline$[\mathrm{M}-2 \mathrm{H}]^{2-}$ & 1076.97 & 1077.04 & \\
\hline$[\mathrm{M}+\mathrm{Na}-3 \mathrm{H}]^{2-}$ & 1087.95 & 1088.03 & \\
\hline$[\mathrm{M}+2 \mathrm{Na}-4 \mathrm{H}]^{2-}$ & 1098.92 & 1099.02 & \\
\hline$[\mathrm{M}-3 \mathrm{H}]^{3-}$ & 723.75 & 723.70 & GT1 (t18:0/20:0) \\
\hline$[\mathrm{M}+\mathrm{Na}-3 \mathrm{H}]^{2-}$ & 1096.93 & 1097.04 & \\
\hline$[\mathrm{M}+\mathrm{Na}-3 \mathrm{H}]^{2-}$ & $1094.95^{*}$ & 1095.04 & GT1 (d18:1/21:0) \\
\hline$[\mathrm{M}-3 \mathrm{H}]^{3-}$ & 727.11 & 727.04 & GT1 (d18:1/22:0) \\
\hline$[\mathrm{M}+\mathrm{Na}-3 \mathrm{H}]^{2-}$ & 1101.92 & 1102.05 & \\
\hline$[\mathrm{M}+\mathrm{Na}-3 \mathrm{H}]^{2-}$ & $1108.92^{*}$ & 1109.06 & GT1 (d18:1/23:0) \\
\hline$[\mathrm{M}+\mathrm{Na}-3 \mathrm{H}]^{2-}$ & 1114.96 & 1115.06 & GT1 (d18:1/24:1) \\
\hline$[\mathrm{M}-3 \mathrm{H}]^{3-}$ & 722.39 & 722.35 & O-Ac-GT1 (d18:1/18:0) \\
\hline$[\mathrm{M}-3 \mathrm{H}]^{3-}$ & 731.74 & 731.70 & O-Ac-GT1 (d18:1/20:0) \\
\hline$[\mathrm{M}-2 \mathrm{H}]^{2-}$ & 1128.95 & 1129.05 & Fuc-GT1 (d18:1/17:0) \\
\hline
\end{tabular}


Table 1. (continued)

$m / z$ (monoisotopic)

\begin{tabular}{lcrl} 
Type of molecular ion & Detected & Calculated & Assigned structure \\
\hline \hline$[\mathrm{M}-2 \mathrm{H}]^{2-}$ & 1144.89 & 1145.06 & Fuc-GT1 (t18:0/18:0) \\
{$[\mathrm{M}-2 \mathrm{H}]^{2-}$} & 1159.89 & 1159.08 & Fuc-GT1 (t18:0/20:0) \\
{$[\mathrm{M}-3 \mathrm{H}]^{3-}$} & 805.40 & 805.38 & GQ1 (d18:1/18:0) \\
{$[\mathrm{M}+\mathrm{Na}-4 \mathrm{H}]^{3-}$} & 812.73 & 812.71 & \\
{$[\mathrm{M}+2 \mathrm{Na}-4 \mathrm{H}]^{2-}$} & 1230.43 & 1230.56 & \\
{$[\mathrm{M}+3 \mathrm{Na}-5 \mathrm{H}]^{2-}$} & 1241.43 & 1241.55 & \\
{$[\mathrm{M}-3 \mathrm{H}]^{3-}$} & 814.74 & 814.72 & GQ1 (d18:1/20:0) \\
{$[\mathrm{M}+\mathrm{Na}-4 \mathrm{H}]^{3-}$} & 822.07 & 822.05 & \\
{$[\mathrm{M}+2 \mathrm{Na}-4 \mathrm{H}]^{2-}$} & 1244.42 & 1244.57 & O-Ac-GQ1 (d18:1/18:0) \\
{$[\mathrm{M}-3 \mathrm{H}]^{3-}$} & $819.38^{*}$ & 819.38 & \\
{$[\mathrm{M}+\mathrm{Na}-4 \mathrm{H}]^{3-}$} & $826.73^{*}$ & 826.71 & \\
\hline
\end{tabular}

$\mathrm{d}=$ dihydroxy sphingoid base; $\mathrm{t}=$ trihydroxy sphingoid base; * low intensity ions

The monoisotopic $\mathrm{m} / \mathrm{z}$ values of ions are given.

17:0), Fuc-GT1 (t18:0/18:0) and Fuc-GT1 (t18:0/20:0). Three extended ganglioside structures attributed to GQ1 are detected as triply and doubly charged ions. The triply charged ion at $m / z 819.38$ accompanied by its sodiated form at $m / z 826.73$ is assigned to O-acetylated GQ1 (d18: 1/18:0) form (Table 1).

\section{Automated Chip-Based NanoESI-QTOF MS/MS in Data-Dependant Acquisition for Ganglioside Fragmentation}

To investigate the possibility of performing rapid and accurate ganglioside sequencing, by the automated MS to MS/MS switching function available on the QTOF mass spectrometer, the triply charged ion at $m / z 708.39$ was selected. This ion is assigned to GT1 (d18:1/18:0). In data-dependant acquisition (DDA), the automated precursor ion selection is set in the "included masses only" option. The specific NanoMate parameters are set in ChipSoft, at $1.67 \mathrm{kV}$ for the nanoESI voltage and 0.45 p.s.i. nitrogen head pressure. The QTOF MassLynx parameters are set at $135 \mathrm{~V}$ for the sampling cone, with $50 \mathrm{eV}$ collision energy during the MS/MS scanning. Figure 3 shows the automated MS/MS data between 280-1600 m/z of the triply charged ion $m / z$ 709.03, which corresponds to GT1 (d18:1/18:0). In our previous work on ganglioside fragmentation by capillary-based (-)nanoESI-QTOF, with manual selection for MS/MS, an extended signal acquisi-

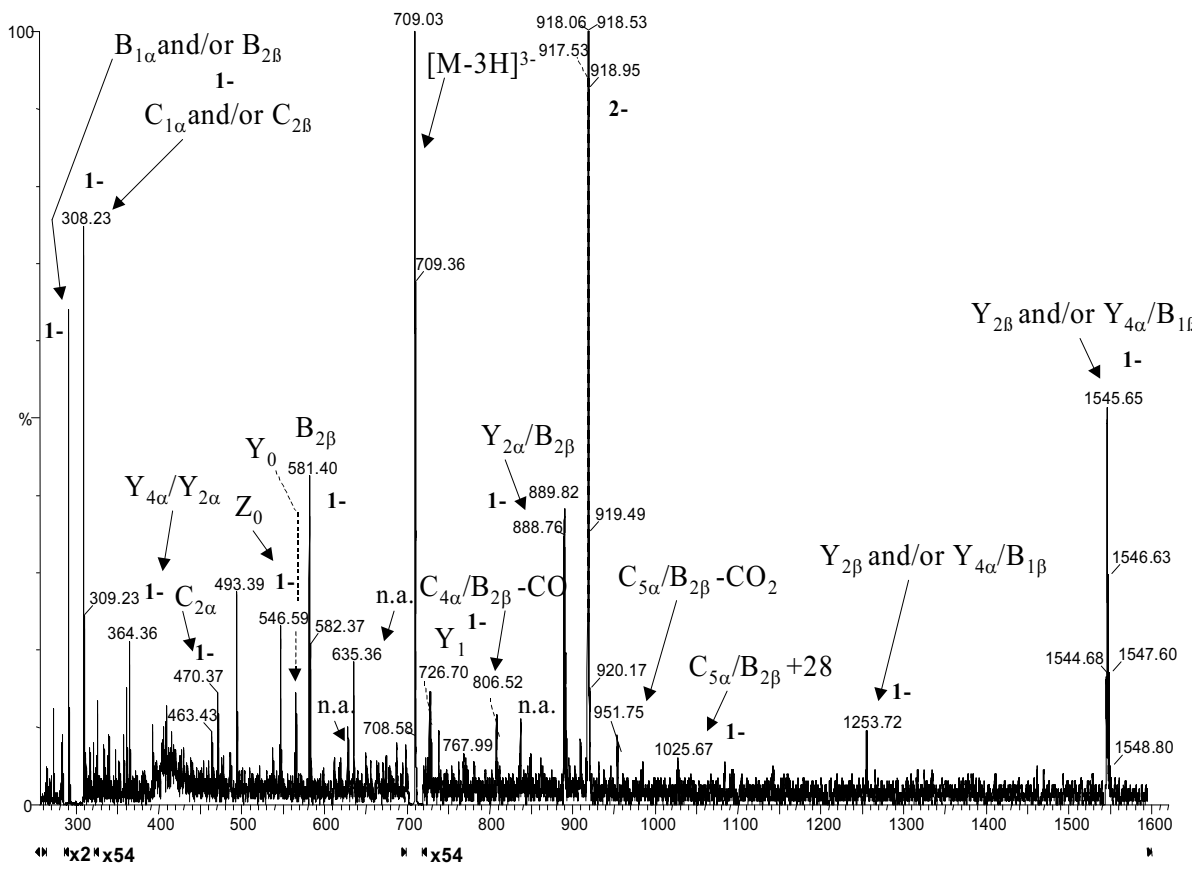

Figure 3. Automated (-)nanoESIchip-QTOF auto MS/MS of the GT1 species detected as a triply charged ion at $\mathrm{m} / \mathrm{z}$ 709.03; cone potential $135 \mathrm{~V}$; collision energy $50 \mathrm{eV}$; acquisition time $1 \mathrm{~min}$. Assignment of fragment ions is according to Domon and Costello [34]. n.a. $=$ not assigned. 


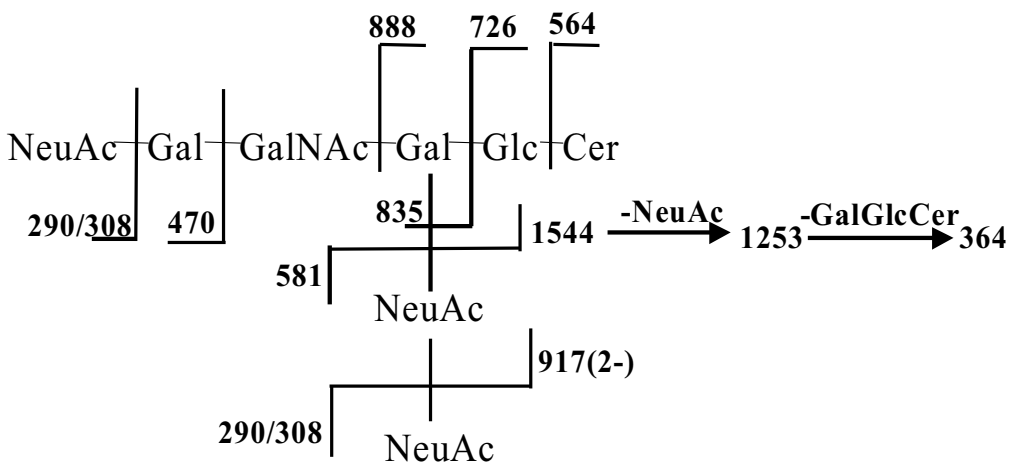

Scheme 1. Fragmentation pathway by autoMS/MS of the GT1 species detected as a triply charged ion at $m / z$ 709.03. The structure depicted refers to GT1b known as dominant isomer in human brain $[27,30]$.

tion time, with a sample consumption of about 50 pmol, was necessary. This was because longer time was required to obtain sufficient diagnostic fragment ions for the structural assignment of GT1 from a purified fraction [30]. In the current study with the nanoESIchip and automatic fragmentation, within $1 \mathrm{~min}$ of acquiring MS/MS data, approximately $0.5 \mathrm{pmol}$ of material were sprayed. When examined, with respect to the acquisition time, sample complexity, and sample consumption, the MS/MS spectrum has a fair signal-to-noise ratio. It also has a sufficient set of "fingerprint" ions for the structural elucidation of the molecule. Abundant GD1 and GM1 fragment ions, which correspond to successive desialylation of the GT1 precursor ion are observed at $m / z 917.53$ (doubly charged) and at $\mathrm{m} / \mathrm{z} 1544.68$ (singly charged), as Y-type ions. The GD1 fragment ion is accompanied by its $B_{1}$ and $C_{1}$ counterparts. They are observed as singly charged ions at $\mathrm{m} / \mathrm{z} 290.20$ and 308.23, respectively. The GM1 fragment ion is accompanied by the singly charged afferent $\mathrm{B}_{2}$ counterpart at $m / z 581.40$, assigned to $\mathrm{NeuAc}_{2}{ }^{-}$. Cleavage of the ceramide moiety forms the singly charged $Y_{0}$ ion at $\mathrm{m} / \mathrm{z}$ 564.75 , and a singly charged $Z_{0}$ ion at $m / z$ 546.50. These fragment ions, in conjunction with the singly charged ion at $m / z 726.70$ (GlcCer) $^{-}$and $m / z 888.76$ (GalGlcCer) $^{-}$are indicative of the presence of the (d18:1/18:0) ceramide residue. Additional supporting information for this fragmentation pathway depicted in Scheme $\mathbf{1}$ is provided by the monosialo fragment ions at $m / z 470.25$ and $m / z$ 835.21, and the GalGalNAc ${ }^{-}$ion at $m / z$ 364.36.

\section{Conclusions}

This study was designed to assess the potential use of the fully automated (-)nanoESIchip-QTOF MS and automated MS/MS for the screening and sequencing of this particular glycoconjugate category. The gangliosides are known to require specific conditions for ionization and detection, fragmentation in tandem MS experiments at low energies. This automated nanoESIchip-QTOF MS methodology, optimized for ganglioside analysis, has provided new insights into their structural diversity, and complex molecular architec- ture. The approach was applied in the analysis of a native mixture of gangliosides, extracted from human cerebellar gray matter. The sample concentration and instrument parameters were revised, in consideration of the particular requirements of the chip-based electrospray ionization process. When compared with capillary-based ESI MS, chip-based ESI MS had greater sensitivity, and gave a clearer representation of the sample composition. NanoESIchip MS screening enabled the identification of 46 glycoforms, with a high degree of heterogeneity in the ceramide motifs and biologically relevant modifications. When fully automated chipESI MS infusion was combined with automated precursor ion selection and fragmentation, a complete structural data set was obtained for single ganglioside species from a native mixture of great complexity. The data was achieved with a short analysis time, and with drastically reduced sample consumption. The fully automated chip-based (-)nanoESI MS approach described here is of general applicability in ganglioside analysis, and represents a step forward in the implementation of automated chipMS methods in glycomics.

\section{Acknowledgments}

The authors thank Dr. Milovan Kubat, forensic pathologist at the University of Zagreb, Croatia, for the brain sample. The financial support of this work was provided by Deutsche Forschungsgemeinschaft within Sonderforschungsbereich 492, project Z2 to JPK. The ESI-QTOF mass spectrometer was obtained from the HbfG grant (Land Nordrhein Westfalen) to JPK. ŽV was supported at the University of Münster by the Deutscher Akademischer Austauschdienst (DAAD) fellowship in 2000.

\section{References}

1. Thorsen, T.; Maerkl, S. J.; Quake, S. R. Microfluidic Large-Scale Integration. Science 2002, 298, 580-584.

2. Groisman, A.; Enzelberger, M.; Quake, S. R. Microfluidic Memory and Control Devices. Science 2003, 300, 955-958.

3. Dethy, J. M.; Ackermann, B. L.; Delatour, C.; Henion, J. D.; Schultz, G. A. Demonstration of Direct Bioanalysis of Drugs in Plasma Using Nanoelectrospray Infusion from a Silicon Chip 
Coupled with Tandem Mass Spectrometry. Anal. Chem. 2003, $75,805-811$.

4. Zhang, S.; Van Pelt, C. K.; Wilson, D. B. Quantitative Determination of Noncovalent Binding Interactions Using Automated Nanoelectrospray Mass Spectrometry. Anal. Chem. 2003, 75, 3010-3018.

5. Van Pelt, C. K.; Zhang, S.; Fung, E.; Chu, I.; Liu, T.; Li, C.; Korfmacher, W. A.; Henion, J. A Fully Automated Nano-Electrospray Tandem Mass Spectrometric Method for Analysis of Caco-2 Samples. Rapid Commun. Mass Spectrom. 2003, 17, 15731578.

6. Benkestock, K.; Van Pelt, C. K.; Akerud, T.; Sterling, A.; Edlun, P. O.; Roeraade, J. Automated Nano-Electrospray Mass Spectrometry for Protein-Ligand Screening by Noncovalent Interaction Applied to Human H-FABP and A-FABP. J. Biomol. Screen. 2003, 8, 247-256.

7. Zhang, S.; Van Pelt, C. K.; Henion, J. D. Automated ChipBased Nano-Electrospray Mass Spectrometry for Rapid Identification of Proteins Separated by Two-Dimensional Gel Electrophoresis. Electrophoresis 2003, 24, 3620-3632.

8. Zaia, J. Mass Spectrometry of Oligosaccharides. Mass Spectrom Rev. 2004, 23, 161-227.

9. Maček, B.; Hofsteenge, J.; Peter-Katalinić, J. Direct Determination of Glycosylation Sites in O-Fucosylated Glycopeptides Using Nano-Electrospray Quadrupole Time-of-Flight Mass Spectrometry. Rapid Commun. Mass Spectrom. 2001, 15, 771-777.

10. Hofsteenge, J.; Huwiler, K. G.; Maček, B.; Hess, D.; Lawler, J.; Mosher, D. F.; Peter-Katalinić, J. C-Mannosylation and OFucosylation of the Thrombospondin Type 1 Module. J. Biol. Chem. 2001, 276, 6485-6498.

11. Sagi, D.; Peter-Katalinić, J.; Conradt, H. S.; Nimtz, M. Sequencing of Tri- and Tetra-antennary N-Glycans Containing Sialic Acid by Negative Mode ESI QTOF Tandem MS. J. Am. Soc. Mass Spectrom. 2002, 13, 1138-1148.

12. Robbe, C.; Capon, C.; Coddeville, B.; Michalski, J. C. Diagnostic ions for the rapid analysis by nano-electrospray ionization quadrupole time-of-flight mass spectrometry of O-glycans from human mucins. Rapid Commun. Mass Spectrom. 2004, 18, 412-420.

13. Zamfir, A.; Seidler, D. G.; Kresse, H.; Peter-Katalinić, J. Structural Characterization of Chondroitin/Dermatan Sulfate Oligosaccharides from Bovine Aorta by Capillary Electrophoresis and Electrospray Ionization Qquadrupole Time-of-Flight Tandem Mass Spectrometry. Rapid Commun. Mass Spectrom. 2002, 16, 2015-2024.

14. Zamfir, A.; Seidler, D. G.; Kresse, H.; Peter-Katalinić, J. Structural Investigation of Chondroitin/Dermatan Sulfate Oligosaccharides from Human Skin Fibroblast Decorin. Glycobiology 2003, 13, 733-742.

15. Wuhrer, M.; Koeleman, C. A.; Deelder, A. M.; Hokke, C. H. Normal-Phase Nanoscale Liquid Chromatography-Mass Spectrometry of Underivatized Oligosaccharides at Low-Femtomole Sensitivity. Anal. Chem. 2004, 76, 833-838.

16. Froesch, M.; Bindila, L.; Zamfir, A.; Peter-Katalinić, J. Sialylation Analysis of $O$-Glycosylated Sialylated Peptides from Urine of Patients Suffering from Schindler's Disease by Fourier Transform Ion Cyclotron Resonance Mass Spectrometry and Sustained Off-Resonance Irradiation Collision-Induced Dissociation. Rapid Commun. Mass Spectrom. 2003, 17, 2822-2832.

17. Zamfir, A.; Vakhrushev, S.; Sterling, A.; Niebel, J.; Allen, M.; Peter-Katalinić, J. Fully Automated Chip-Based Mass Spectrometry for Complex Carbohydrate System Analysis. Anal. Chem. 2004, 76, 2046-2054.

18. Zamfir, A.; Peter-Katalinić, J. Glycoscreening by On-Line Sheathless Capillary Electrophoresis/Electrospray Ionization-
Quadrupole Time-of-Flight Tandem Mass Spectrometry. Electrophoresis 2001, 22, 2448-2457.

19. Schnaar, R. L.; Collins, B. E.; Wright, L. P.; Kiso, M.; Tropak, M. B.; Roder, J. C.; Crocker, P. R. Myelin-Associated Glycoprotein Binding to Gangliosides. Structural Specificity and Functional Implications. Annu. N.Y. Acad. Sci. 1998, 845, 92-105.

20. Hakomori, S.; Handa, K. Interaction of Glycosphingolipids with Signal Transducers and Membrane Proteins in Glycosphingolipid-Enriched Microdomains. Methods Enzymol. 2003, 363, 191-207.

21. Svennerholm, L.; Boström, K.; Fredman, P.; Månson, J. E.; Rosengren, B.; Rynmark, B. M.; Human Brain Gangliosides: Developmental Changes from Early Fetal Stage to Advanced Age. Biochim. Biophys. Acta 1989, 1005, 109-117.

22. Kračun, I.; Rösner, H.; Drnovsek, V.; Vukelić, Z.; Cosović, Č.; Trbojević-Čepe, M.; Kubat M. Gangliosides in the Human Brain Development and Aging. Neurochem. Int. 1992, 20, 421-431.

23. Ariga, T.; Jarvis, W. D.; Yu, R. K. Role of SphingolipidMediated Cell Death in Neurodegenerative Diseases. J. Lipid Res. 1998, 39, 1-16.

24. Kračun, I.; Rösner, H.; Čosovic, Ć.; Stavljenić, A. Topographical Atlas of the Gangliosides of the Adult Human Brain. J. Neurochem. 1984, 43, 979-989.

25. Svennerholm, L.; Boström, K.; Jungbjer, B.; Olsson, L. Membrane Lipids of Adult Human Brain: Lipid Composition of Frontal and Temporal Lobe in Subjects of Age 20 to 100 Years. J. Neurochem. 1994, 63, 1802-1811.

26. Santiago, M. F.; Costa, M. R.; Mendez-Otero, R. Immunoblockage of 9-O-acetyl GD3 Ganglioside Arrests the in Vivo Migration of Cerebellar Granule Neurons. J. Neurosci. 2004, 24, 474-478.

27. Vukelić, Z.; Metelmann, W.; Müthing, J.; Kos, M.; PeterKatalinić, J. Anencephaly: Structural Characterization of Gangliosides in Defined Brain Regions. Biol Chem. 2001, 382, 259-274.

28. O'Connor, P. B.; Budnik, B. A.; Ivleva, V. B.; Kaur, P.; Moyer, S. C.; Pittman, J. L.; Costello, C. E. A High Pressure MatrixAssisted Laser Desorption Ion Source for Fourier Transform Mass Spectrometry Designed to Accommodate Large Targets with Diverse Surfaces. J. Am. Soc. Mass Spectrom. 2004, 15, $128-132$.

29. Colsch, B.; Afonso, C.; Popa, I.; Portoukalian, J.; Fournier, F.; Tabet, J. C.; Baumann, N. Characterization of the Ceramide Moieties of Sphingoglycolipids from Mouse Brain by ESI-MS/ MS: Identification of Ceramides Containing Sphingadienine. J. Lipid Res. 2004, 45, 281-286.

30. Metelmann, W.; Vukelić, Z.; Peter-Katalinić, J. Nano-Electrospray Ionization Time-of-Flight Mass Spectrometry of Gangliosides from Human Brain Tissue. J. Mass Spectrom. 2001, 36, 21-29.

31. Zamfir, A.; Vukelić, Z.; Peter-Katalinić, J. A Capillary Electrophoresis and Off-Line Capillary Electrophoresis/Electrospray Ionization-Quadrupole Time-of-Flight Tandem Mass Spectrometry Approach for Ganglioside Analysis. Electrophoresis 2002, 23, 2894-2903.

32. Peter-Katalinić, J. Analysis of Glycoconjugates by Fast Atom Bombardment Mass Spectrometry and Related MS Techniques. Mass Spectrom. Rev. 1994, 13, 77.

33. Schultz, G. A.; Corso, T. N.; Prosser, S. J.; Zhang, S. A Fully Integrated Monolithic Microchip Electrospray Device for Mass Spectrometry. Anal. Chem. 2000, 72, 4058-4063

34. Domon, B.; Costello, C. A Systematic Nomenclature for Carbohydrate Fragmentations in FAB-MS/MS Spectra of Glycoconjugates. Glycoconj. J. 1988, 5, 397-409. 